\title{
Two-phase electrolysis process modelling: from the bubble to the electrochemical cell scale
}

\author{
P. Mandin ${ }^{1}$, H. Roustan ${ }^{2}$, R. Wüthrich ${ }^{3}$, J. Hamburger ${ }^{4}$ \\ \& G. Picard ${ }^{1}$ \\ ${ }^{1}$ LECA UMR 7575 CNRS-ENSCP-Paris 6, ENSCP, Paris Cedex, France \\ ${ }^{2}$ Alcan, Centre de Recherche de Voreppe, Voreppe Cedex, France \\ ${ }^{3}$ E.P.F.L. - L.S.R. - CH-1015 Lausanne, Suisse \\ ${ }^{4}$ Transoft International, Fluidyn, Saint-Denis, France
}

\begin{abstract}
During two-phase electrolysis for hydrogen, aluminium or fluor production, there are bubbles which are created at electrodes which imply a great hydrodynamic acceleration but also quite important electrical properties and electrochemical processes disturbance. There are few works concerning the local modelling of electrochemical processes during a two-phase electrolysis process. Nevertheless, effects like the anode effect, particularly expensive on the point of the process efficiency, should need a better understanding. The goal of the present work is to present the modelling and the numerical simulation of the gas production, from the single bubble scale to the macroscopic electrochemical cell, during the two-phase electrolysis process. Bubbles are motion sources for the electrolysis cell flow, and then hydrodynamic properties are strongly coupled with species transport and electrical performances. The presence of bubbles modifies these global and local properties: the electrolysis cell and the current density distribution are modified. The present work shows theoretical modelling on both scales and also performance changes during the two-phase electrolysis processes.
\end{abstract}

Keywords: two-phase electrolysis modelling electrochemical bubbles.

\section{Introduction}

Gas release and induced fluid flow over electrodes exist in many electrochemical processes such as aluminium, fluorine production, water electrolysis and many 
other electrochemical processes. The hydrodynamic properties and the gas-flow motion in electrochemical cells are of great practical interest in electrochemical engineering science since the dispersed phase modifies the electrical properties of the electrolyte (as well as mass and heat transfers), and therefore modifies the macroscopic cell performances. In many cases, this phenomenon has to be avoided, but, in some other processes, the gas flow rate has to be controlled. This control is a great challenge, a multi scale one. The macroscopic performances are local gas concentration dependent, with a strong coupling with hydrodynamic and electric properties; but the gas volume shape and size are dependent of bubble scale properties, but also of the local hydrodynamic shear stress. The bubble formation during electrochemical processes and its electrochemical cell scale consequences is a few investigated fields [1-7], whereas the same topic has been intensively explored for heat transfer science or gas injection through porous walls for turbulence promotion and transfer increase [8-10].

Nevertheless the strong knowledge of the coupled phenomena is necessary: it is the case for electrochemical processes such as for gas production $\left(\mathrm{CO}_{2}, \mathrm{~F}_{2}, \mathrm{H}_{2}\right.$, $\left.\mathrm{O}_{2}, \mathrm{Cl}_{2}, \ldots\right)$ [1-6] and other processes such as, for example, the chemical engraving (SPARK) [7].

In all these electrochemical processes, a coupling effect is particularly strong because bubble-dispersed phase acts like an electrical shield, the shielding effect depending on the density of the bubbles, which is namely the local gas volume fraction of the dispersion.

The present work shortly presents experimental set-up, bubble scale modelling and electrochemical cell scale modelling. The vertical gas evolving electrode configuration is more developed and primary current distribution evolution with electrolysis applied potential is presented and compared with experimental results.

\section{Experimental set-up}

A rectangular electrolysis cell (height $=10 \mathrm{~cm}$, length $=8 \mathrm{~cm}$, width $=5 \mathrm{~cm}$ ) has been chosen. The investigated working electrode was made with: a platinum pin, a tin oxide $\mathrm{SnO}_{2}$ deposit upon a plane glass sample, a carbon one, and a tungsten one. Potassium hydroxide $\mathrm{KOH} 1 \mathrm{mM}$ is added in the water as support salt and the final $\mathrm{pH}$ was 11 . The alkaline water electrolysis is considered here as a reference process for the general two-phase electrolysis:

$$
\text { Anode: } \mathrm{OH}^{-}=0.25 \mathrm{O}_{2}+0.5 \mathrm{H}_{2} \mathrm{O}+\mathrm{e}^{-}, \mathrm{E}^{\circ}=0.401 \mathrm{~V} / \mathrm{NHE}
$$

The counter electrode is a tungsten wire put in a separated compartment (isolated from the electrolyte by means of sintered glass) to study only one electrode induced two-phase flow. The reference electrode used is the silver one put in a sheath of glass filled with a $\mathrm{KCl} 3 \mathrm{M}$ solution, $\mathrm{AgCl}$ saturated. Electrochemical measurements (cyclic voltammetry, chrono amperometry and impedance spectroscopy) are performed with a Parstat 2273. The visualization, movies and photographs are performed with BIPOL Y11P camera completed with a modular endoscope H.S.W with illumination by halogen lamp. 


\section{The single bubble: from appearance at the electrode to departure from the electrode}

In this part a single "in formation" bubble is considered alone in a motionless environment.

\subsection{The bubble appearance}

It is considered here one single electrochemical reaction, cathode or anode, leading first to a dissolved gas and second to bubbles nucleation. At the beginning the electrochemical product is first dissolved in the electrolyte if the electrolyte is not already saturated with dissolved gas. In fact beginning period is really difficult to model because of this uncertainty on the liquid electrolyte. For example it is known that there is always nitrogen and oxygen dissolved gas in laboratory electrolyte and then, because of the oxidizing capacity of oxygen, it is necessary to eliminate these atmospheric gas with, for example, the use of an argon flow before experiment. These attentions are usual in laboratory cells but not at industrial scale! Nevertheless, after this electrolyte preparation period, the criterion for the appearance of the first two-phase character is related to the thermodynamic Henry law: $X_{k}=P_{k} / H$. H is the Henry constant for the dissolved gas, electrolyte and considered temperature; $P_{k}$ is the partial pressure of species $\mathrm{k}$ whereas $X_{k}$ is the maximum molar fraction of dissolved gas k. For example, $\mathrm{H}_{\mathrm{O} 2}\left(20^{\circ} \mathrm{C}\right)=4.0610^{9} \mathrm{~Pa}$, and then, before the use of argon flow, $\mathrm{P}_{\mathrm{O} 2}=0.2110^{5}$ $\mathrm{Pa}$, and the electrolyte has an oxygen molar fraction $\mathrm{X}_{\mathrm{O} 2}\left(20^{\circ} \mathrm{C}\right)=5.2410^{-6}$. But to create pure oxygen bubbles, the partial pressure in equilibrium with electrolyte must be $\mathrm{P}_{\mathrm{O} 2}=10^{5} \mathrm{~Pa}$ and then $\mathrm{X}_{\mathrm{O} 2}$ must reach $2.4610^{-5}$ at electrode to satisfy this thermodynamic criterion.

The Henry constant increases with temperature; for example $\mathrm{H}_{\mathrm{O} 2}\left(50^{\circ} \mathrm{C}\right)=$ $5.9610^{9} \mathrm{~Pa}$. It is interesting here, in the case of the water electrolysis process study, to give the Henry constant values for hydrogen: $\mathrm{H}_{\mathrm{H} 2}\left(50^{\circ} \mathrm{C}\right)=6.9210^{9} \mathrm{~Pa}$ and $\mathrm{H}_{\mathrm{H} 2}\left(50^{\circ} \mathrm{C}\right)=7.7510^{9} \mathrm{~Pa}$. These values are associated to pure water. But, often, electrochemical processes use support salt to increase electrical conductivity and reduce ohmic drop. In the condition of a large ionic strength Is $=0.5 \Sigma_{k}\left(z_{k}^{2} \cdot C_{k}\right)$, with $\mathrm{z}_{\mathrm{k}}$ the charge of ion $\mathrm{k}$ and $\mathrm{C}_{\mathrm{k}}$ its composition $\left(\mathrm{mol} \mathrm{l}^{-1}\right)$, the Henry constant is modified [11]. In the present case Is $=1 \mathrm{M}$ and $\mathrm{h}=0.134$ [11], so $\mathrm{H}_{\mathrm{O} 2} / \mathrm{H}_{\mathrm{O} 2 \text {,ref }}=0.73$. When the steady regime is reached, the dissolved species $\mathrm{k}$ composition at electrode is supposed to be constant and equal to the thermodynamic value.

The nucleation phenomenon, at the very beginning, and the transient description is difficult because of the initial conditions, the possibility of nonthermodynamic phenomena like over saturation, and the necessity to know the flow at the electrode vicinity. It is also difficult when a steady state regime is observed. If at the very beginning there is no flow, transport is purely diffusive and then $\mathrm{k}_{\mathrm{OH}}=\mathrm{D}_{\mathrm{OH}} / \delta(\mathrm{t})$, the $\mathrm{OH}^{-}$species transport velocity at electrode is fast: when $\mathrm{t} \rightarrow 0, \delta(\mathrm{t}) \rightarrow 0$. $\mathrm{D}_{\mathrm{OH}}$ is the diffusion coefficient and $\delta(\mathrm{t})$ is the unsteady boundary layer thickness given by the Cottrell theory. Then the nucleation 
criterion is reached fast in absence of forced flow. If a forced flow is used (Couette flow, RDE or IRDE flows), the transport law is different and the nucleation phenomenon disappears. It can be only in small flow zones (for example nearthe electrode axis for a RDE configuration). In absence of electrochemically induced flow, the steady transport velocity is: $\mathrm{k}_{\mathrm{OH}}=\mathrm{D}_{\mathrm{OH}} / \delta$, with the constant boundary layer thickness $\delta$ (for example $\delta=1.61 \mathrm{D}^{1 / 3} \cdot \omega^{-1 / 2} \cdot v^{1 / 6}$ for the $\mathrm{RDE}$ configuration). At the steady regime, for a constant applied current density, it can be written for the applied current density $\mathrm{j}\left(\mathrm{A} \mathrm{m}^{-2}\right)$ :

$$
\begin{aligned}
& \mathrm{j} /(\mathrm{nF})=\mathrm{k}_{\mathrm{OH}} \cdot \rho_{\text {electrolyte }} / \mathrm{M}_{\text {electrolyte}} \cdot\left(\mathrm{X}_{\mathrm{OH}, \text { bulk }}-\mathrm{X}_{\mathrm{OH}, \mathrm{el}}\right) \\
& =4 \mathrm{k}_{\mathrm{O} 2} \cdot \rho_{\text {electrolyte }} / \mathrm{M}_{\text {electrolyte }} \cdot\left(\mathrm{X}_{\mathrm{O} 2, \mathrm{el}}-\mathrm{X}_{\mathrm{O} 2 \text {,bulk }}\right)
\end{aligned}
$$

The effective mass transport kinetic constant is called $\mathrm{k}_{\mathrm{OH}}$ and $\mathrm{k}_{\mathrm{O} 2}$ respectively for the reactive and product; $\rho_{\text {electrolyte }}$ and $\mathrm{M}_{\text {electrolytet }}$ are respectively the density and the molar weight of the electrolyte; $n$ is the electron number exchanged in reaction (here $\mathrm{n}=1$ ) and the Faraday constant is $\mathrm{F}=96500 \mathrm{C} \mathrm{mol}^{-1}$. A strong flow allows large limiting current density but has no incidence upon the dissolved oxygen molar fraction at electrode $\mathrm{X}_{\mathrm{O} 2 \text {,el }}$. For these forced flow study, the bubble nucleation is not evident if the oxygen bulk composition is zero $\mathrm{X}_{\mathrm{O} \text {, bulk }}=0$, in case for example of argon gas use. Even if a forced flow is not used, a flow is electrochemically induced due to the departure of bubbles and their motion exchange with electrolyte. It is really difficult after this bubble flow installation to know the effective transport velocity $\mathrm{k}$, which is no longer the purely diffusive one or the forced flow one. This electrochemically induced flow is particularly strong for vertical or horizontal "floor" electrodes but almost negligible for horizontal "ceiling" electrodes. The electrode position and direction must be directly related to the gravity field direction.

It is also observed that the nucleation sites obey a regular mesh. It has also been observed that the nucleation process always take place at the same place. Though the high difficulty of this process study, there is some help from nature!

\subsection{The bubble growth}

A single bubble is here considered. The study of electrochemical bubble growth can use analogy with other scientific works: the gas injection through a porous surface or the phase change of a coolant flow for example in vapour generator or heat exchangers $[1-2 ; 8-10]$. The use of these other science works allows a better understanding but the analogy must be limited. Gas injection is often used to promote turbulence and heat or mass transfer. In this case, the gas flow is totally fixed parameter. In heat transfer, the analogy with the two-phase electrolysis is better but also different. If the primary flow is highest Reynolds number, the heat flux Q (W) is imposed in the same manner as the intensity I (A) is imposed, because the thermal resistance due to the metal separating primary and secondary flows is generally the smaller one. The gas at the electrode and in the electrode vicinity has a screening effect, interfacial (effective area for the electrical current reduced according with dimensionless screened area $\alpha$, figure 1) and homogeneous (because of void fraction $\varepsilon$, thermodynamic and kinetic transport properties are modified, flow modified, etc) because the conducting 
electrolyte is replaced with gas, an insulator. It is also true in a reduced way for heat transport: the gas has an about 10 times smaller heat conduction coefficient and then it also screens the interface and modifies the flow properties. The analogy is strong for the research of primary current distribution. But for secondary or ternary current distribution the analogy is worse. Because the current density is increased due to the actual electro active area, the over potential are larger. Because the reactive electro active species arrive at electrode with about the same rate as the product species leave the place: the interface properties are then strongly coupled with homogeneous transport.

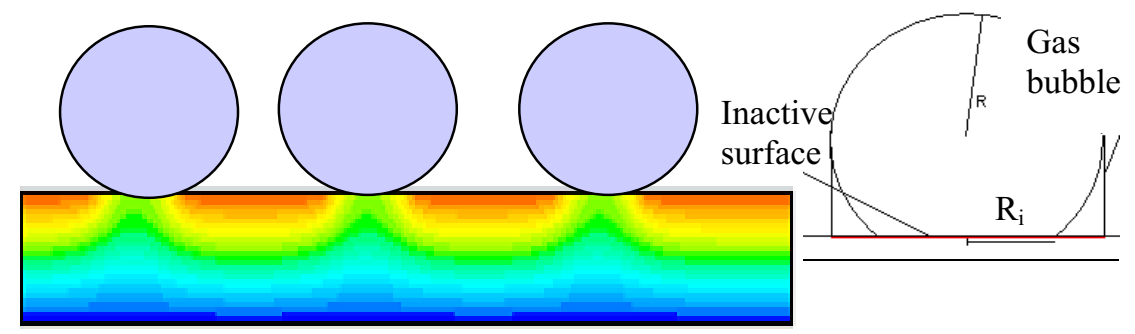

Figure 1: Current density contour in electrode due to bubble screening effect.

Until the dissolved gas concentration at electrode has reached the nucleation criterion, the first gas domains are observed at spatially periodical situated nucleation sites. The growth occurs in both normal to electrode and tangential to electrode directions. The growth kinetic is faster, like for crystals growth, in the tangential to electrode, the radial one. It is because, first; the dissolved species concentration is maximum at this electrode plane. This is also because the coalescence forces are confronted to the flow stress in the normal to electrode direction. So the gas domain should first be more developed in the radial direction from the nucleation site than in the normal direction. The volume is, at this time, defined as a plane disk shape. The electrode area is partially screened (figure 1), and the gas domain feeding can't be from the electrode contact area. The inactive area seems experimentally to be usually constant in location.

Then the growth of the gaseous volume continues but the mass exchange is performed with the unsteady contact area with electrolyte. Because the imposed current is constant, though the reduced electro active area, the effective applied current density $\mathrm{j}$ at the electrode-electrolyte contact area (called free area) increases and is $j_{\text {eff }}=\mathrm{j} /(1-\alpha)$. The oxygen production continues at this free area. The dissolved gas molar fraction is generally supposed the saturation one (thermodynamic hypothesis), but the value can be larger because of the increasing current density and because over saturation processes can occur. Under steady regime assumption, this electrode dissolved oxygen production must be consumed by transport and gas domain growth at nucleation sites phenomena.

To know the rhythm of gas domain volume increase it is necessary to know both the intensive mass transport flux from liquid electrolyte to the gaseous

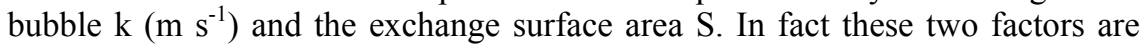


related because the unsteady bubble shape modifies the local flow and then the mass exchange.

The convecto-diffusive mass transport velocity is calculated from empirical correlation for the Sherwood number $\mathrm{Sh}=\mathrm{k}_{\mathrm{O} 2} \cdot \mathrm{d} / \mathrm{D}_{\mathrm{O} 2}$. For a free spherical bubble (diameter $\mathrm{d}$ ) in a uniform flow with relative velocity $\mathrm{V}_{\mathrm{r}}$, the correlation is: $\mathrm{Sh}=$ $2+0.66 \operatorname{Re}^{0.5} \cdot \mathrm{Sc}^{0.3}$, for $10<\operatorname{Re}<10^{4}$, with relative Reynolds number $\mathrm{Re}=\rho \cdot \mathrm{V}_{\mathrm{r}} \cdot \mathrm{d} / \mu$, $\rho$ and $\mu$ are the electrolyte density $\left(\mathrm{kg} \mathrm{m}^{-3}\right)$ and viscosity $(\mathrm{Pa} . \mathrm{s})$ respectively. $\mathrm{Sc}=$ $\mu /\left(\rho . \mathrm{D}_{\mathrm{O} 2}\right)$ is the Schmidt number. Other correlation $\mathrm{Sh}=2+0.79 \mathrm{Re}^{0.5} \mathrm{Sc}^{1 / 3}$ is also used by Vogt et al $[1-2 ; 8]$, using an average velocity at the anchored at electrode bubble. In fact it is difficult to use rigorously these kinds of correlations: First because an internal circulation is possible inside the bubble volume. In this case correlations must take into account the gas viscosity and also the surface tension $\gamma$ (which is $73 \mathrm{mN} \mathrm{m}^{-1}$ for water against air at $20^{\circ} \mathrm{C}$ ). Second, because at electrode, with bubbles, the flow can't be uniform and simple, it seems to be better to introduce the local wall shear stress $\tau_{\mathrm{p}}=\mu . \partial \mathrm{V}_{\text {tangential }} / \partial$ normal $(\mathrm{Pa})$ or the hydrodynamic stress $\mathrm{s}=\partial \mathrm{V}_{\text {tangential }} / \partial$ normal $\left(\mathrm{s}^{-}\right.$ $\left.{ }^{1}\right)$. But the interaction between such a stressed flow and the growing bubble is a "to explore" situation under steady and unsteady assumptions. The local wall shear stress is also related to the entire cell flow, because of the elliptic nature of Navier-Stokes equations. The bubble shape is also an important parameter: it changes with the bubble feeding and characteristic length $\mathrm{d}$ increase. Nevertheless, the molar feeding rate is $<\mathrm{k}_{\mathrm{O} 2}(\mathrm{t})>$. $\mathrm{S}(\mathrm{t}) \cdot\left(\mathrm{C}_{\mathrm{O} 2 \text {,flow }}-\mathrm{C}_{\mathrm{O} 2 \text {,bubble }}\right)$. It is difficult to estimate this flux potential which implies both heterogeneous and homogeneous Henry saturation concentration.

The growing bubble shape is also difficult to describe. One important property is the contact angle $\theta$ which is determined with the triplet gaselectrolyte-electrode. For a given gas and a given electrolyte, this contact angle can be large or small. This contact angle property is generally related to the surface tension $\gamma\left(\mathrm{N} \mathrm{m}^{-1}\right)$. These relatively well-known properties are nevertheless static properties in stagnant electrolyte. During the two-phase electrolysis, the flow, forced or purely induced, due to bubble, modify the bubble shape. Duhar and Colin have shown the modification of the anchoring angle in a Couette flow. Duluc et al have also shown the evolution with time of this angle. Then the actual unsteady anchored bubble shape is still an unknown only experimentally accessible with movie image processing.

Then, growing bubbles are supposed to obey an unsteady force equilibrium, between:

- the vertical Archimede force: $\left(\rho_{\text {electrolyte }}-\rho_{\text {gas }}\right) \cdot g$. Vol, with $\mathrm{Vol}=\pi \cdot \mathrm{h}^{2} \cdot(\mathrm{d} / 2-\mathrm{h} / 3)$ and $\mathrm{g}=9.81 \mathrm{~m} \mathrm{~s}^{-2}$.

- the flow direction friction force: $\iint_{\text {wet }} \boldsymbol{\tau}_{\mathrm{P}} . \mathrm{d} \mathbf{S}=<\tau_{\mathrm{P}}>$.Surf, with Surf $=\pi$.h.(2.d-h); $<\boldsymbol{\tau}_{\mathrm{P}}>$.is a difficult to access property directly related to the flow friction at the bubble wet surface.

- the capillarity friction force: $\int_{\text {contact }} \gamma . \mathrm{dl}=\left\langle\gamma>\right.$.L, with $\mathrm{L}=2 . \pi . \mathrm{d} .[\mathrm{h} / \mathrm{d} .(1-\mathrm{h} / \mathrm{d})]^{1 / 2}$; $<\gamma>$.is about $\gamma$ the surface tension in the considered case.

Because the bubble shape is unsteady, the contact angle is unsteady $[1-2 ; 8-$ $10]$ and these three forces are also unsteady. 


\subsection{The bubble "takeoff" and its replacement}

At the growth beginning these three forces sum is dominated by the capillarity friction. But, when the bubble dimension increases, the Archimede and hydrodynamic forces increase faster than the capillarity one [1-2; 11-12]. So, for a given dimension $\mathrm{d}$, these two forces begin larger than the contact one. The detachment can occur at the electrode contact but can also occur elsewhere at the bubble volume frontier. Duhar and Duluc works [9-10] show that the detachment does not occur at the electrode but due to a critical elongation and finally the rupture phenomenon. Then, at the electrode, a gaseous volume is still present which also accumulate the neighbouring dissolved oxygen. The phenomenon takes place in the same place because of this begun nucleation.

The takeoff criterion is difficult to establish though it is important for the evolved bubble size prediction. In fact it is sure that detachment occurs for forces larger than the capillary one. But after this, a bubble surface elongation occurs and the rupture occurs only after.

\subsection{The bubble displacement in the electrolyte}

The single bubble now presented has leaved the electrode surface and its life now consists in rejoining the free surface of the electrolyte according with the gravity field $\mathrm{g}=9.81 \mathrm{~m} \mathrm{~s}^{-2}$.

The bubble is supposed to be instantaneously a sphere with the diameter $\mathrm{d}$ verifying:

$$
\pi \cdot \mathrm{d}^{3} / 6=\text { last anchored bubble volume Vol }
$$

This sphere is considered rigid, with a constant diameter if:

$$
\mathrm{d}<\left(\gamma /\left(\left(\rho_{\text {electrolyte }}-\rho_{\text {gas }}\right) \cdot \mathrm{g}\right)^{1 / 2}\right.
$$

Under this assumption, the Newton law for the single bubble in the electrolyte is:

$$
\text { M.dV/dt }=\Sigma \text { Forces }
$$

\subsubsection{Vertical velocity}

$$
\begin{gathered}
\mathrm{dV}_{\text {gas }} / \mathrm{dt}=\text { Archimede }+ \text { friction } \\
=\left(\rho_{\text {electrolyte }} / \rho_{\text {gas }}-1\right) \cdot g- \pm 6 / 8 \cdot \rho_{\text {electrolyte }} / \rho_{\text {gas }} \cdot C_{\mathrm{x}} / \mathrm{d} \cdot\left(\mathrm{V}_{\text {electrolyte }}-\mathrm{V}_{\text {gas }}\right)^{2}
\end{gathered}
$$

If the bubble velocity $\mathrm{V}_{\text {gas }}$ is larger than the local electrolyte velocity $\mathrm{V}_{\text {electrolyte, }}$, then the friction force is negative (stagnant electrolyte for example). On the other hand, if electrolyte motion is large, then the friction force is a motion source for bubble and the term is positive.

The drag coefficient $C_{x}$ is a function of the Reynolds number $\operatorname{Re}_{\mathrm{p}}$ calculated with the relative velocity; $C_{x}=24 / R_{p}$ for smaller velocities according with $R_{p}$ $<0.1$. The evolution of the drag coefficient $C_{x}$ has been given by Morsi and Alexander [13].

This equation has been solved with an explicit scheme and a time step $1 \mu$ s or $0.5 \mu \mathrm{S}$. When the sum of the forces applied to the bubble is constant, the bubble increases its velocity from zero to $\mathrm{V}_{\mathrm{lim}}$ according with algebraic equation:

$$
\left(1-\rho_{\text {gas }} / \rho_{\text {electrolyte }}\right) \cdot g- \pm 6 / 8 . C_{x} / d .\left(V_{\text {electrolyte }}-V_{\text {gas }}\right)^{2}=0
$$

The limiting relative velocity is strongly diameter dependent whereas the gas chemical nature through $\rho_{\text {gas }}$ is not important. One information very important is that the limiting velocity is reached very quickly, in time lower than $50 \mu$ s, which 
leads to distance smaller than $5 \mu \mathrm{m}$. Then, the bubble vertical relative velocity will be generally supposed constant anywhere in the electrochemical cell. But the electrolyte flow has generally strong changes with space and then with bubble trajectory time. The knowledge of local electrolyte velocity $\mathrm{V}_{\text {electrolyte }}$ is of great importance, but very difficult to model. The electrolyte flow is elliptic, strongly coupled with the electrochemical cell configuration and gas flow dependent. This flow description uses Navier-Stokes modelling and computational fluid dynamic software; it will be presented in next part. Bubbles are motion source for electrolyte.

\subsubsection{Horizontal velocity}

The single bubble obeys essentially vertical forces, Archimede and friction one. But, for large current density and bubble production, mostly for vertical or horizontal ceiling configurations, the bubble trajectory is experimentally not purely vertical. In the horizontal ceiling case, the bubble accumulation at the electrode implies coalescence and surface displacement. It is difficult to mathematically formalize the fact that for a gas evolving vertical electrode, the bubbles can't all stay at the electrode. The two-phase flow develops like a boundary layer, with an increasing thickness from bottom to top. The bubble is then submitted to a horizontal force, not explainable for the bubble alone, but due to interaction with electrode surface and others bubbles. Indeed, if the flow is purely vertical but with gradient (velocity zero at electrode), a slip force (also called Saffman force) is induced from small to large velocity values. This force is in fact a pressure force and the gradient is correctly described with the Bernoulli law. The result of this force is a distance of the bubble from the electrode. There is also interaction of the considered bubble with other bubbles: chocks or also pressure and wake effects. These interactions can imply coalescence phenomena. It is to notice here that the previous part has shown the small inertia of a single bubble. The limiting velocity given previously is relative with the local electrolyte velocity. Then, the more the bubble is far from the electrode (with electrolyte velocity zero) the faster and pressure effect is induced in this direction. There are few modelling attempts of these phenomena. Some models used the classical molecular interaction modelling in term of force $\mathbf{F}_{\mathrm{h}}$ and associated potential field $\psi: \mathbf{F}_{\mathrm{h}}=-\operatorname{grad}(\psi)$ with $\psi=-\mathrm{K}[\mathrm{d} / \mathrm{x}]^{\mathrm{n}}$. The potential law introduces two unknown constants $K$ and $n$, and the parameter $d / x$, with $d$ the bubble diameter and $\mathrm{x}$ the distance from electrode. Krepper [14] has also corrected and developed the Antal semi-empirical model, also with this molecular interaction analogy. This difficulty is the reason why the vertical gas evolving electrode is more difficult to describe than the horizontal floor one. The horizontal ceiling configuration, like in the classical Hall-Héroult configuration, is particular because phenomena are essentially horizontal on surface.

\section{From the single bubble to the macroscopic two-phase electrolysis modelling}

The previous part has shown that it is difficult to know the actual two-phase hydrodynamic flow property and particularly the boundary layer thickness in 
case of vertical electrode. However, this knowledge is important for the local properties (thermodynamic and transport) evaluation and then the macroscopic scale electrolysis modelling. This is the reason why a lot of authors are interested in horizontal floor gas evolving electrode configuration, like the IRDE (Inverted Rotating Disk Electrode). The following part presents electrolysis cell performance modelling, with first a reduced model and second the use of computational fluid dynamic software for electrolyte flow calculation.

\subsection{Reduced model}

Before heavy calculations with commercial computational software it is interesting to evaluate some easy to access properties of the two-phase electrolysis process. It is easy to calculate how many bubbles of a given diameter $\mathrm{d}$ are created per unit time for an imposed intensity $\mathrm{I}(\mathrm{A}) \mathrm{I}=\mathrm{I} . \mathrm{S}$, with $\mathrm{j}$ the local current density $\left(\mathrm{A} \mathrm{m}^{-2}\right)$ and $\mathrm{S}$ the electro active area; $\mathrm{j} /(\mathrm{n} . \mathrm{F})$ is the local gas molar rate production; R.T/P is the molar volume of an ideal gas (such as $\mathrm{O}_{2}$ or $\mathrm{H}_{2}$ ) with pressure $\mathrm{P}=1.01310^{5} \mathrm{~Pa}$, temperature $\mathrm{T}=300 \mathrm{~K}$ and gas constant $\mathrm{R}=8.314 \mathrm{~J}$ $\mathrm{mol}^{-1} \mathrm{~K}^{-1} ; \pi . \mathrm{d}^{3} / 6$ is the bubble volume. The created bubble rate is then:

$$
\mathrm{dN} / \mathrm{dt}=6 . I . R . T /\left(\text { n.F.P. } \pi \cdot \mathrm{d}^{3}\right)\left(\text { evolved bubbles } \mathrm{s}^{-1}\right)
$$

The calculation of the electrolysis cell performances needs the knowledge of configuration and dimensions of cell and electrodes.

\subsubsection{Horizontal floor electrode}

The evolving electrode is supposed to be horizontal at the cell floor. Then the bubble trajectory is purely vertical if bubble interaction with other bubbles or cells boundaries is neglected. If the electrolyte height is $\mathrm{H}$, the bubble, supposed to have instantaneously the limiting velocity $V_{\text {lim }}$, will have the residence time $\tau$ $=/ \mathrm{V}_{\text {lim }}$ in the cell. After a fast transition, it can be supposed that the bubble number in the cell is steady and obeys:

$$
\mathrm{F}=\mathrm{dN} / \mathrm{dt} . \tau=6 . I \cdot R \cdot T \cdot H /\left(\text { n.F.P. } \pi \cdot \mathrm{d}^{3} \cdot \mathrm{V}_{\mathrm{lim}}\right)
$$

To simplify, the configuration is supposed one-dimensional (1D). The electrolyte height change $\mathrm{H}^{\prime}-\mathrm{H}$ due to the steady bubbles presence in the liquid domain:

$$
\mathrm{H}^{\prime} / \mathrm{H}=1+\mathrm{j} . \mathrm{R} . \mathrm{T} /\left(\text { n.F.P. } \mathrm{V}_{\text {lim }}\right)
$$

This property is important because it is an easy to access experimental evidence but need very sensitive liquid level measurements: for a $1 \mathrm{~mm}$ diameter bubble, the relative elevation is lower than $2.5 \%$ for a $\mathrm{j}=1 \mathrm{~A} \mathrm{~cm}^{-2}$ imposed current density. The volume change is generally neglected. One important parameter is also the void fraction $\varepsilon$ :

$\varepsilon=$ bubbles steady volume/cell volume $=\mathrm{j} . \mathrm{R} . \mathrm{T} /\left(\right.$ n.F.P. $\left.\mathrm{V}_{\text {lim }}\right)=\left(\mathrm{H}^{\prime}-\mathrm{H}\right) / \mathrm{H}$

In the same way the void fraction is lower than $2.5 \%$ for current density $j=1 \mathrm{~A}$ $\mathrm{cm}^{-2}$.

The ohmic resistance of the electrolyte is then modified due to the presence of this insulating volume. This resistance is electrodes configuration dependent but also electrical conductivity $\sigma$ dependent. The generally used relation is the Bruggeman one for a two-phase electrolyte, with an insulating second phase with small volume fraction $\varepsilon$ :

$$
\sigma=\sigma_{0} \cdot(1-\varepsilon)^{3 / 2}
$$


At the electrolysis beginning the electrolyte is stagnant; but when the steady regime is reached, the electrolyte has won motion from the evolving bubbles:

$$
\begin{aligned}
\mathrm{F}_{\text {electrolyte }} \cdot \mathrm{V}_{\text {electrolyte }} & =\Sigma_{\text {bubbles }} \mathrm{F}_{\text {friction }}=\mathrm{N} \cdot \pi \cdot \mathrm{d}^{2} / 8 \cdot \rho_{\text {electrolyte }} \cdot \mathrm{V}_{\text {lim }}{ }^{2} \cdot \mathrm{C}_{\mathrm{x}}\left(\mathrm{V}_{\text {lim }}\right) \\
& =\text { N. } \pi \cdot \mathrm{d}^{3} / 6 \cdot\left(\rho_{\text {electrolyte }}-\rho_{\text {gas }}\right) \cdot \mathrm{g} \\
& =\text { I.R.T.H. } \rho_{\text {electrolyte }} \cdot \mathrm{g} /\left(\mathrm{n} . \mathrm{F} \cdot \mathrm{P} . \mathrm{V}_{\text {lim }}\right)
\end{aligned}
$$

This vertical motion is given to the electrolyte in contact with bubbles. But, this locally given motion is exchanged in all the electrolyte volume according with the phenomenological law of Fourier, for example for Newtonian electrolytes: $\tau$ $=-\mu \operatorname{grad} \mathrm{V}$, with $\tau$ the motion exchange density vector $(\mathrm{Pa})$. In fact the electrolyte is confined and the flow mass conservation will force the electrolyte flow direction rotation: at the free surface, the bubbles exit the electrolyte but not the electrolyte! And then it has to rotate in the perpendicular direction. Then the electrolyte is stagnant only at the process very beginning but not at steady state; this is the reason why the two experiment families are interesting: unsteady and steady regime experimental measurements. Then, motion is both vertical and horizontal, and the perfect knowledge of the local electrolyte flow is possible to determine only with computational fluid dynamic programs. This will be presented in the last part.

\subsubsection{Vertical electrode}

The vertical electrodes are used for industrial production of gaseous fluorine $\mathrm{F}_{2}$; $\mathrm{H}_{2}$ bubbles are also created at the counter electrode. Like for the classical water electrolysis, both electrodes produce bubbles and it is really difficult to model the anode and cathode induced flows interaction.

Reference [7] shows experimental evolution of the electrochemical cell electrical performances during a two-phase electrolysis process. The imposed potential $U$ is increased from the minimal value $U_{d}$ for electrolysis. For the smaller potential values, the intensity I increase is almost linear (zone from A to B). With the bubbles production increase, the equivalent resistance increase and the linear evolution is lost and the intensity I becomes constant with value $\mathrm{I}^{\text {crit }}$ (zone from $\mathrm{B}$ to $\mathrm{C}$ ). The potential increase leads to no intensity increase till the value $\mathrm{U}^{\text {crit }}$. Between points $\mathrm{C}$ and $\mathrm{D}$, the situation becomes unstable and with a small potential increase from $\mathrm{U}^{\text {crit }}$, the intensity decreases rapidly. At the minimal intensity level, a new process occurs: the tool electrode is heated to a visibly large temperature. Bubbles must be, in this zone (between D and E), electrochemically produced, but also because of electrolyte phase change due to high temperature. Then it appears in this zone sparks. The numerous bubbles show an important electrolyte flow, according with a double vertices structure, at the electrode tip.

The case of the vertical gas evolving electrode is more difficult to model than the horizontal floor one because of the residence time. In the cell, the bubble residence time is no longer the same for bubbles emitted at electrode bottom and top: the residence time is also $\tau$ for the bottom emitted bubbles but it is 0 for top emitted bubbles. Because the limiting velocity is reached instantaneously, if the current density distribution at electrode was uniform, the bubbles steady number should be $\mathrm{N} / 2$. A lot of previously presented properties are usable here. But the 
difficulty is due to the determination of the actual bubbles trajectory, vertical but mostly horizontal. This is because the actual two-phase volume and the associated boundary layer thickness are difficult to know that the ohmic resistance and other macroscopic cells properties are difficult to model (see the previous part the difficulty to describe the horizontal force exerted upon the single bubble).

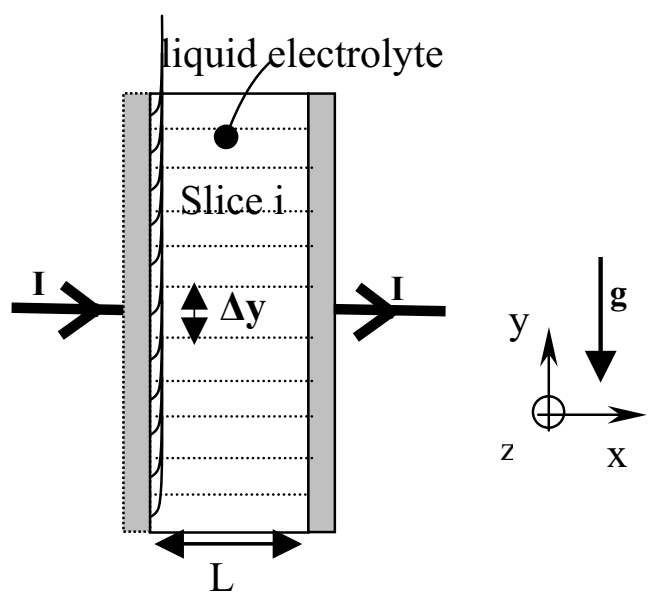

Figure 2: Vertical gas evolving electrode.

Figure 2 shows the vertical gas evolving electrode configuration. The Newman model for vertical electrodes uses a one dimensional vertical discretization and the local bubble number in each horizontal slice. This number is increasing from the bottom where $\mathrm{N}$ is about zero to the top where $\mathrm{N}$ is maximal.

The current density distribution is then clearly not uniform: the current density is maximal at the bottom and minimal at the top, around the average current density $\mathrm{j}_{\mathrm{av}}=\mathrm{I} / \mathrm{S}$. A simple recurrence algorithm, based upon bubble mass balance, has been programmed in $\mathrm{C}$ language to evaluate at slice $\mathrm{i}$, the local vertical bubbles fluxes $\mathrm{F}_{\mathrm{i}}$ and the steady bubbles number $\mathrm{N}_{\mathrm{i}}$ :

$$
F_{i}=F_{i-1}+6 . I_{i} \cdot R \cdot T /\left(n . F . P . \pi . d^{3}\right)
$$

Like in the previous part, the fluxes are immediately obtained. The local bubble number needs more information in term of local bubble velocity which is directly related to the local electrolyte velocity. If the bubbles size and bubbles relative velocity are supposed uniform, it is also possible to calculate the slice $\mathrm{i}$ bubble number, void fraction, resistance and then the primary current distribution under these assumptions. The numerical procedure is iterative.

$$
\mathrm{N}_{\mathrm{i} .}=\mathrm{F}_{\mathrm{i} .} \Delta \mathrm{y} / \mathrm{V}_{\mathrm{i}}=\mathrm{F}_{\mathrm{i}} \cdot \Delta \mathrm{y} / \mathrm{V}_{\mathrm{lim}}
$$

The following results have been obtained supposing an electrolyte conductivity $\sigma_{0}=1 \mathrm{~S} \mathrm{~m}^{-1}$, an electro-active surface $25 \mathrm{~cm}^{2}$ and a two-phase volume with $\mathrm{L}=10^{-2} \mathrm{~m}$. The one phase process has the theoretical resistance $4 \Omega$. 
The height $\mathrm{h}=5 \mathrm{~cm}$ has been dicretized with 100 slices. The bubbles fluxes, bubbles number, local void fraction, local electrical conductivity and local current density profiles have been calculated.

Table 1: Electrochemical cell macroscopic properties evolution with potential.

\begin{tabular}{|c|c|c|c|c|}
\hline $\mathrm{U}_{\text {imposed }}(\mathrm{V})$ & $\mathrm{I}(\mathrm{A})$ & $\mathrm{j}_{\text {average }}\left(\mathrm{A} \mathrm{m}^{-2}\right)$ & Resistance $(\Omega)$ & Elevation $(\mathrm{m})$ \\
\hline $1.00 \mathrm{E}+02$ & $2.45 \mathrm{E}+01$ & $9.79 \mathrm{E}+03$ & 4.09 & $7.10 \mathrm{E}-04$ \\
\hline $1.00 \mathrm{E}+03$ & $1.99 \mathrm{E}+02$ & $7.97 \mathrm{E}+04$ & 5.02 & $7.10 \mathrm{E}-03$ \\
\hline $2.00 \mathrm{E}+03$ & $3.09 \mathrm{E}+02$ & $1.24 \mathrm{E}+05$ & 6.48 & $1.42 \mathrm{E}-02$ \\
\hline $3.00 \mathrm{E}+03$ & $3.49 \mathrm{E}+02$ & $1.39 \mathrm{E}+05$ & 8.61 & $2.13 \mathrm{E}-02$ \\
\hline $3.50 \mathrm{E}+03$ & $3.51 \mathrm{E}+02$ & $1.40 \mathrm{E}+05$ & 9.97 & $2.49 \mathrm{E}-02$ \\
\hline
\end{tabular}

The table 1 gives the calculation results from small imposed potential $U_{\text {imposed }}$ to the critical potential which is here $3550 \mathrm{~V}$ with an associated intensity $\mathrm{I}=351$ A. For imposed potential greater than this value, the void fraction $\varepsilon$ reaches $100 \%$ at the electrode top and the calculation procedure is stopped.

Table 1 shows intensity non-linear increasing with imposed potential. The ohmic resistance increases due to the bubbles presence which also leads to an electrolyte level elevation associated with the steady gas volume in the electrolyte (initial height is $\mathrm{h}=5 \mathrm{~cm}$ ).

In the used algorithm, the critical point $\mathrm{C}$ is defined as the imposed potential which leads to a $100 \%$ gas fraction at the electrode top. But in fact, the current can always flows after this potential value, not at the top but at lower positions. This is not taken into account here and will be in future works.

The default of this model is the use of the geometrical length $\mathrm{L}$ between electrodes instead of the actual two-phase boundary layer thickness, and the simplification of the electrolyte and bubbles local velocities properties.

\subsubsection{Horizontal ceiling electrode}

This case is frequently met for example with the classical rotating disk electrode (RDE). It is also one of the major problems of the Hall-Heroult process for aluminium production; in this case In this case the working electrode is generally very screened due to the bubbles accumulation. It has been shown in the first part dedicated to the single bubble that the Archimede force was important for the bubble take-off from the electrode. Because of this horizontal configuration, this force can't lead to bubble motion, which can also be horizontal. Then friction force from the flow is alone to ensure this bubble motion. Other phenomena must be considered: the "on electrode surface" bubble motion; the meeting of other bubbles and the coalescence. The more the gaseous volume is, the more the height and then the flow friction. Experiments with this configuration are on progress. 


\subsection{Eulerian-Lagrangian model}

As said in previous part, this kind of approach must be improved with a better description of the electrolyte and bubbles flows. But there are few two-phase flow modelling, mostly in the case of a second phase created at the boundary. A lot of works in fluid mechanics or combustion sciences are developed, but the second phase is generally created or injected in the calculation domain, not at the border, in interaction with boundary layers. The flow can also be turbulent: it is generally the case for large motion source and then for large bubbles creation. The Navier-Stokes electrolyte flow modelling is classically written:

Mass balance:

Motion balance:

$$
\partial \rho_{\mathrm{e}} / \partial \mathrm{t}+\partial\left(\rho_{\mathrm{e}} \cdot \mathrm{u}_{\mathrm{i}}\right) / \partial \mathrm{x}_{\mathrm{i}}=0
$$

$$
\partial\left(\rho_{\mathrm{e}} \cdot \mathrm{u}_{\mathrm{i}}\right) / \partial \mathrm{t}+\mathrm{u}_{\mathrm{j} .} \partial\left(\rho_{\mathrm{e}} \cdot \mathrm{u}_{\mathrm{i}}\right) / \partial \mathrm{x}_{\mathrm{j}}=-\partial \mathrm{P} / \partial \mathrm{x}_{\mathrm{i}}+\partial\left(\mu_{\mathrm{e}} \partial \mathrm{u}_{\mathrm{i}} / \partial \mathrm{x}_{\mathrm{i}}\right) / \partial \mathrm{x}_{\mathrm{i}}+\mathrm{K}_{\mathrm{i}}
$$

with $u_{i}$ the $i^{\text {th }}$ component of the local electrolyte velocity; $\rho_{e}$ is the local equivalent density $\left(\mathrm{kg} \mathrm{m}^{-3}\right)$; $\mathrm{P}$ is the local gauge pressure $(\mathrm{Pa})$ and $\mu_{\mathrm{e}}$ is the local equivalent dynamic viscosity (Pa.s). The local flow properties are calculated with computational fluid dynamic software using finite volumes method [15] such as Fluent ${ }^{\circledR}$ or Fluidyn ${ }^{\circledR}$. Equivalent values are used to take into account the two-phase and evenly turbulent local flow character. The calculation of these equivalent thermodynamic and transport properties need to consider single bubble and group of bubbles: the equivalent density is directly related to the void fraction $\varepsilon: \rho_{\mathrm{e}}=\rho_{0} .(1-\varepsilon)$ whereas the equivalent viscosity must be calculated for a given hydrodynamic stress and a given void fraction. $\mathrm{K}_{\mathrm{i}}$ is the motion source term due to bubbles Archimede acceleration. The coupling between bubbles motion and this electrolyte motion source is calculated for each cells of the calculation domain mesh. The effort from the flowing bubbles to the electrolyte is mostly due to the sum of the friction terms over all the possible trajectories and the associated bubbles crossing the considered cell:

$$
\mathrm{K}_{\mathrm{i}}=\Sigma_{\text {trajectories }} \text { friction.q. } \Delta \mathrm{t} / \text { Volume }_{\text {cell }}
$$

with friction the acceleration previously presented $\left(\mathrm{m} \mathrm{s}^{-2}\right)$; q the bubble mass flow of one considered trajectory $\left(\mathrm{kg} \mathrm{s}^{-1}\right)$ and $\Delta \mathrm{t}$ and Volume cell $_{\text {the residence }}$ time (s) and the volume $\left(\mathrm{m}^{3}\right)$ of the considered cell respectively. Each electrode discretization faces are associated with a bubbles emitted trajectory, or more precisely, its gravity centre. In the case of the vertical electrode configuration, if no horizontal force is introduced, these trajectories are exactly at the electrode which leads to quick accumulation problem. This is the reason why a horizontal dispersion force must be introduced to take into account the two-phase boundary layer thickness increase. Numerical results previously published [16-17] have shown that this horizontal force must be about half the Archimede one order of range to allow a good accordance with Tobias experimental results [5] obtained with a segmented vertical electrode.

To calculate primary current distribution, the usual CFD codes equations must be completed with an electric charge balance equation such as:

$$
\partial\left(\sigma_{\mathrm{e}} \partial \varphi / \partial \mathrm{x}_{\mathrm{i}}\right) / \partial \mathrm{x}_{\mathrm{i}}=0
$$


with $\sigma_{\mathrm{e}}=\sigma_{0} .(1-\varepsilon)^{3 / 2}\left(\mathrm{~S} \mathrm{~m}^{-1}\right)$ according with Bruggeman law and $\varphi$ the local potential (V).

This is generally easy to add diffusion equations in these commercial softwares.

This model is under the dispersed phase assumption: it must be used only for void fraction lower than $20 \%$. This is the reason why the Eulerian-Eulerian model is generally preferred.

\subsection{Eulerian-Eulerian model}

The Eulerian-Eulerian two-phase flow model also uses both the mass and motion balances but for each phase:

Liquid mass balance:

Liquid motion balance:

$$
\partial \rho_{1} / \partial t+\partial\left(\rho_{1} \cdot u_{i, 1}\right) / \partial x_{i}=0
$$

$$
\partial\left(\rho_{1} \cdot u_{i, 1}\right) / \partial t+u_{i, l} \cdot \partial\left(\rho_{1} \cdot u_{i, 1}\right) / \partial x_{j}=-\partial P / \partial x_{i}+\partial\left(\mu_{1} \partial u_{i, 1} / \partial x_{i}\right) / \partial x_{i}+K_{i}
$$

Gas mass balance:

Gas motion balance:

$$
\partial \rho_{\mathrm{g}} / \partial \mathrm{t}+\partial\left(\rho_{\mathrm{g}} \cdot \mathrm{u}_{\mathrm{i}, \mathrm{g}}\right) / \partial \mathrm{x}_{\mathrm{i}}=0
$$

$$
\partial\left(\rho_{\mathrm{g}} \cdot \mathrm{u}_{\mathrm{i}, \mathrm{g}}\right) / \partial \mathrm{t}+\mathrm{u}_{\mathrm{i}, \mathrm{g}} \cdot \partial\left(\rho_{\mathrm{g}} \cdot \mathrm{u}_{\mathrm{i}, \mathrm{g}}\right) / \partial \mathrm{x}_{\mathrm{j}}=-\partial \mathrm{P} / \partial \mathrm{x}_{\mathrm{i}}+\partial\left(\mu_{\mathrm{g}} \partial \mathrm{u}_{\mathrm{i}, \mathrm{g}} / \partial \mathrm{x}_{\mathrm{i}}\right) / \partial \mathrm{x}_{\mathrm{i}}-\mathrm{K}_{\mathrm{i}}
$$

$\mathrm{K}_{\mathrm{i}}$ is the motion exchange between gas phase and liquid phase due to friction, lift, added mass or pressure variation. The local void fraction for a given cell in the mesh leads to the bubble number evaluation and then to the number of friction forces exchanged between the two phases. This model is rigorous but really heavier: four non-linear equations, strongly coupled and the convergence numerical conditions are generally difficult to found. Simplified two-phase flow models are generally proposed in computational fluid dynamic software: the "Mixture Model" (or "Algebraic Slip Model", ASM) and the "Volume Of Fluid" (VOF) model. The first one is mostly used for homogeneous gaseous phase with volume fraction from $10 \%$ to $50 \%$, whereas the second is mostly dedicated for unsteady-free surface problems. This model can be used in the case of really large gaseous structures in the electrolyte, with clearly defined two-phase interface.

\section{Conclusion}

The two-phase electrolysis processes are of a great interest for industrial applications. They not yet have been rigorously modelled though it is now possible to compute the coupling between electrochemistry and two-phase flows. The present work has presented macroscopic and bubble scale modelling and has shown that both scales are coupled which define a multi-scale process. Bubble scales results have to be correlated to lead to a predictive model usable at the larger scales. The present work has focused its attention upon the vertical gas evolving electrode and has shown modification of primary current distribution in case of electrochemically induced two-phase flow. Future works will develop the strong coupling between phenomena and scales and between experimental evidences and calculations. 


\section{References}

[1] Vogt H. and Balzer R.J., The bubble coverage of gas-evolving electrodes in stagnant electrolytes. Electrochimica Acta, 50, 10, pp. 2073-2079, 2005.

[2] Eigeldinger J. and Vogt H., The bubble coverage of gas-evolving electrodes in a flowing electrolyte. Electrochimica Acta, 45, 27, pp. 44494456, 2000.

[3] Javit A. Drake, Clayton J. Radke and Newman J., Transient linear stability of a Simons-process gas-liquid electrochemical flow reactor using numerical simulations. Chemical Engineering Science, 56, 20, pp. 58155834, 2001.

[4] Hine F., Murakami M. J. Electrochem. Society., Vol 127, p. 292, 1980.

[5] Tobias C.W., The influence of attached bubbles on Potential Drop and Current Distribution at Gas-Evolving Electrodes. J. El. Soc., 134, 2, 1959.

[6] Mandin Ph., Hamburger J., Bessou S. and Picard G., Modelling and calculation of the current density distribution evolution at vertical gasevolving electrodes. Electro. Acta, 51, 6, pp. 1140-1156, 2005.

[7] Wüthrich R. and Bleuler H., A model for electrode effects using percolation theory. Electro. Acta, Volume 49, Issues 9-10, Pages 15471554, 2004.

[8] Vogt H., Aras Ö. and Balzer R.J., The limits of the analogy between boiling and gas evolution at electrodes. I. J. Heat Mass Trans., 47, 4, p. 787, 2004.

[9] Duhar G., Colin C. C. R. Mecanique 331, 2003.

[10] Duluc M.-C., Stutz B. and Lallemand M., Transient nucleate boiling under stepwise heat generation for highly wetting fluids. I. J. Heat Mass Trans., 47, 25, p. 5541, 2004.

[11] Janssen L.P.B.M., Warmoeskerken M.M.C.G., Transport Phenomena Data Companion, Arnold DUM, 1987.

[12] Devos O., Gabrielli C. and Tribollet B., Nucleation-growth process of scale electrodeposition-Influence of the mass transport. Elec.a Acta, 52, 1, p.285, 2006.

[13] Morsi S. A. and Alexander A. J., An Investigation of Particle Trajectories in Two-Phase Flow Systems. J. Fluid Mech., 55(2), pp. 193-208, 1972.

[14] Krepper E., Lucas D. and Prasser H.-M., On the modelling of bubbly flow in vertical pipes. Nuclear Engineering and Design, 235, 5, p. 597, 2005.

[15] Patankar S. V., Numerical Heat Transfer and Fluid Flow, Taylor \& Francis, 1980.

[16] Kiss L., Ponscak S., Effect Of Bubble Growth Mechanism On The Spectrum Of Voltage Fluctuations In The Reduction Cell, Light Metals, 2002.

[17] Janssen L.J.J., Behaviour Of Mass Transfer At Gas-Evolving Electrodes, Electrochemica Acta, Vol. 34, No 2, pp. 161-169, 1989. 